\title{
University of Southern California Norris Comprehensive Cancer Center
}

National Cancer Institute

\section{Source}

National Cancer Institute. University of Southern California Norris Comprehensive Cancer

Center. NCI Thesaurus. Code C39556.

The University of Southern California Norris Comprehensive Cancer Center is committed to investigating the complex origins and progression of cancer, developing treatment and prevention strategies, and searching for cures. The Center's research is organized into thematic programs focusing on molecular genetics, epigenetics and regulation, tumor microenvironment, cancer epidemiology, and cancer control research; and translational programs concentrating on genitourinary cancers, gastrointestinal cancers, women's cancers, leukemia and lymphoma, and developmental therapeutics. It was named an $\mathrm{NCl}$-designated comprehensive cancer center in 1973. 